\title{
Construcción del paisaje en áreas de montaña. Arquitectura popular y explotación del territorio en el municipio de Gistaín
}

\author{
Carlos Fernández Piñar \\ ${ }^{a}$ Doctorando ETSAM, C/ Reyes, 9, 28015 Madrid. carlosfpinar@hotmail.com
}

\begin{abstract}
Resumen
En este trabajo se analizan los paisajes resultado de las actividades económicas tradicionales en un entorno de montaña. Se trata del municipio oscense de Gistaín (Chistén), situado en el extremo noroccidental de la comarca de Sobrarbe en Huesca.

Este municipio, dentro del valle del mismo nombre, conservó unos sistemas de exlotacion tradicionales evolucionados a lo largo de varios siglos hasta el último cuarto del siglo XX. Entre las actividades desarrolladas destacaron por su impronta en el paisaje la agrícola y la ganadera, esta última tanto en su vertiente ovina trashumante como en la del ganado mayor trasterminante. La interacción entre todas ellas, acompasando los ciclos agrícolas y los ganaderos, así como la posición geográfica del municipio (al mismo tiempo cerrada al exterior y abierta a influencias diversas, entre ellas las de la vertiente francesa de la cordillera) dotan a sus paisajes de una fuerte personalidad dentro del conjunto del Pirineo aragonés.
\end{abstract}

Por otro lado, y dentro de una interpretación del paisaje y del territorio como patrimonio cultural, se estudian las formas arquitectónicas que sirvieron de soporte a estas actividades económicas, tanto agrícolas como pastoriles, como parte fundamental de la construcción del paisaje.

Palabras clave: paisaje cutural, arquitectura popular, Pirineos, pastoralismo, agricultura de montaña.

\begin{abstract}
This paper analyzes the landscapes resulting from traditional economic activities in a mountain environment. It is the Huesca municipality of Gistain (Chistén), located in the extreme northwest of the Sobrarbe region.

This municipality, within the valley of the same name, preserved some traditional systems evolved over several centuries until the last quarter of the 20th century, so that its footprint is still very present in the landscape. Among the activities carried out, agricultural and livestock farming stood out for their imprint on the landscape. The interaction between these activities, accompanying the agricultural and livestock cycles, as well as the geographical position of the municipality (at the same time closed to the exterior and open to diverse influences, including those of the French side of the mountain range) endow its landscapes with a strong personality within the whole of the Aragonese Pyrenees.

On the other hand, and within an interpretation of the landscape and the territory as cultural heritage, the architectural forms that supported these economic activities, both agricultural and pastoral, as a fundamental part of the construction of the landscape are studied.
\end{abstract}

Keywords: cultural landscape, vernacular architecture, Pyrenees, pastoralism, mountain agriculture. 


\section{Introducción. Situación}

El municipo de Gistaín se encuentra en el extremo NO de la comarca de Sobrarbe del Pirineo aragonés. Se trata de un territorio enclavado entre altos macizos montañosos y con accesos difíciles, especialmente desde el Sur, hasta la apertura de las carreteras modernas. Esta circunstancia y su posición geográfica en un entorno de transición entre distintos ámbitos culturales, fronterizo con los valles de la vertiente francesa y límitrofe con la comarca de la Ribagorza, propiciaron una relación más intensa con estos territorios, que se refleja en unos modelos de asentamiento y unos sistemas de explotación del territorio particulares que se extienden también por el valle de Bielsa y que difieren notablemente de los empleados en la mayor parte de la comarca a la que pertenece. Mientras en la mayor parte de esta el modelo de asentamiento se basa en una multiplicidad de pequeñas aldeas muy próximas entre sí y en el disfrute mancomunado de los pastos estivales, el municipio de Gistaín cuenta con un único núcleo poblacional que administró en exclusividad sus puertos y colonizó su entorno en base a una dispersión de construcciones auxiliares por todo el término.

Los sistemas de explotación agroganadera y las arquitecturas a ellos asociadas han sido estudiadas mediante la combinación del análisis de la documentación histórica existente, (con especial atención a los amillaramientos de los años 1854, 1863 y 1879), la información oral recogida de sus últimos usuarios y el trabajo de campo con elaboración de fichas de inventario de todas las construciones.

\section{Uso tradicional del territorio}

Una primera cuestión necesaria es aclarar el significado y alcance que damos al concepto de lo tradicional. Ni las formas de explotación, ni los sistemas agrícolas y ganaderos empleados, ni tampoco las arquitecturas asociadas han sido en ningún caso invariables en el tiempo, sino que han evolucionado de forma acompasada a los cambios económicos y sociales. La organización agropastoril que nos disponemos a exponer tiene su origen con toda probabilidad en la Baja Edad Media, cuando se conformaron las grandes rutas trashumantes entre las montañas del Pirineo y el valle del Ebro.

Resultado de estas actividades podemos contemplar el paisaje actual, que entendemos como un archivo histórico de las formas de vida y de los sistemas de explotación del territorio. Entre estas actividades han sido las agropecuarias las que han tenido una mayor incidencia en la construcción del paisaje, junto a las actividades extractivas, tanto mineras como forestales.

Las características más destacadas de la transformación antrópica de los territorios de montaña por parte de las sociedades tradicionales son, por un lado, su carácter extensivo a la totalidad del término propio; por otro, la gradación en el espacio y en el tiempo de todos los aprovechamientos humanos.

En la Figura 1 se muestra el término municipal de Gistaín. Dentro de él los espacios tanto agrícolas como ganaderos se escalonaban en una serie de conjuntos diferenciados por su distancia al núcleo de población, por su altitud y por el periodo y la intensidad de su utilización. El espacio productivo agrícola del municipio se organizaba en una serie de conjuntos sucesivamente más alejados del pueblo, con características diferentes. De mayor a menor proximidad a las casas eran (Fig. 1):

- Los huertos. Situados junto a las casas y en pequeñas parcelas regadas junto a los barrancos próximos.

- Los campos primarios alrededor del núcleo de población. Eran destinados a la producción de cereales, patatas y legumbres, incluyendo también algunos prados.

- Los cultivos en el fondo de valle del río Cinqueta, cerrado y estrecho. En las zonas en que las condiciones lo permitían aparecían tanto campos de cultivo como prados de siega. Las parcelas agrícolas ascendían también por las partes bajas de las laderas de los barrancos subsidiarios.

- Los panares constituían un siguiente escalón en la distribución territorial de los cultivos. Se trataba de zonas destinadas al cultivo de cereal situadas a considerable altitud en partidas más alejadas del pueblo, ocupando las partes altas de las laderas de los barrancos auxiliares. Estaban sujetas a una regulación comunal que hacía posible su uso también como zonas de pasto para los ganados. 


\section{Fernández Piñar}

En cuanto al espacio ganadero, los pastos del municipio de Gistaín quedaban ordenados en dos conjuntos claramente diferenciados en función de su altitud, su lejanía respecto del núcleo y sobre todo en función de su periodo de empleo dentro del ciclo anual. Se distinguían los pastos intermedios o de tránsito y los de verano. Los primeros se denominaban habitualmente bajantes, se encuentran en contacto con las fincas particulares de los panares y constituían un paso intermedio en el periplo del ganado desde los pastos de invernada hasta los pastos estivales. Se empleaban, por tanto, en la primavera y el otoño. Los segundos se denominan genéricamente montañas o puertos,y su periodo de uso iba desde finales de julio hasta mediados de septiembre (Fig. 1).

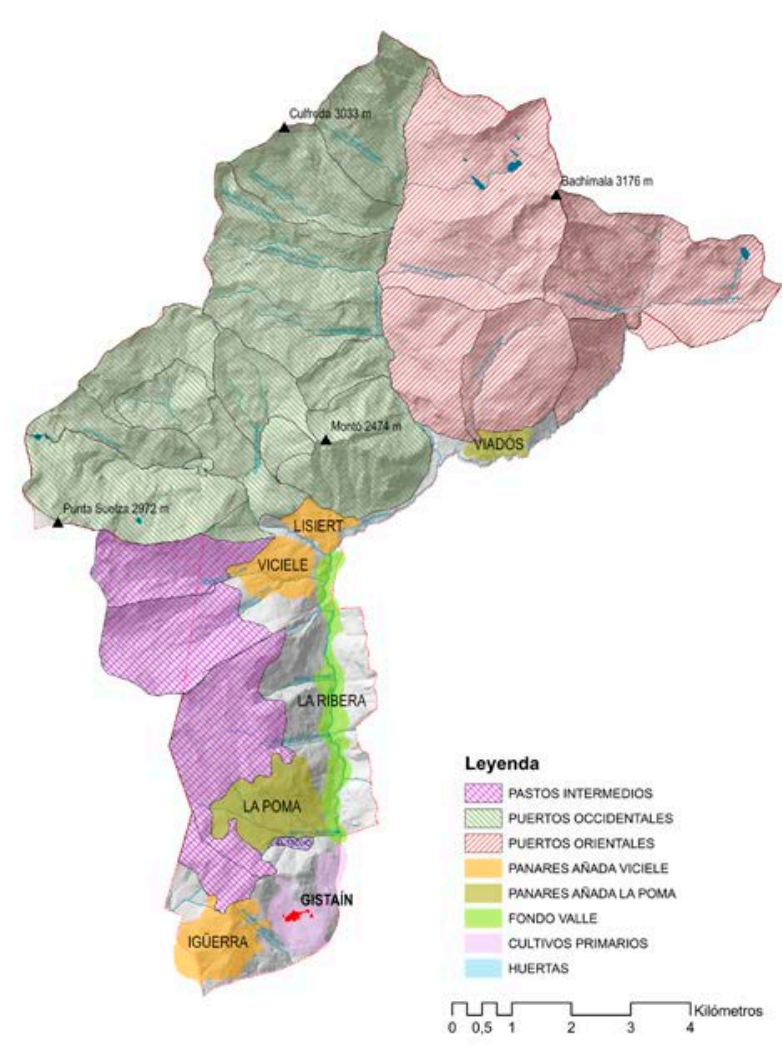

Fuente: Elaboración propia

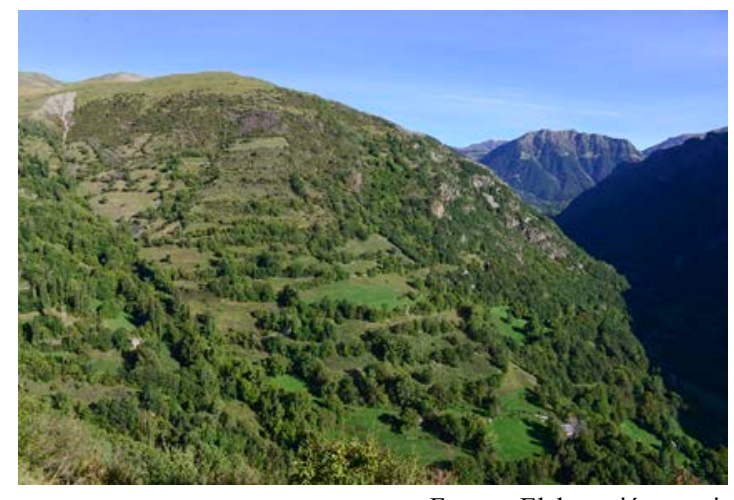

Fuente: Elaboración propia

Fig. 2 Bocage en la parte baja del sector La Poma

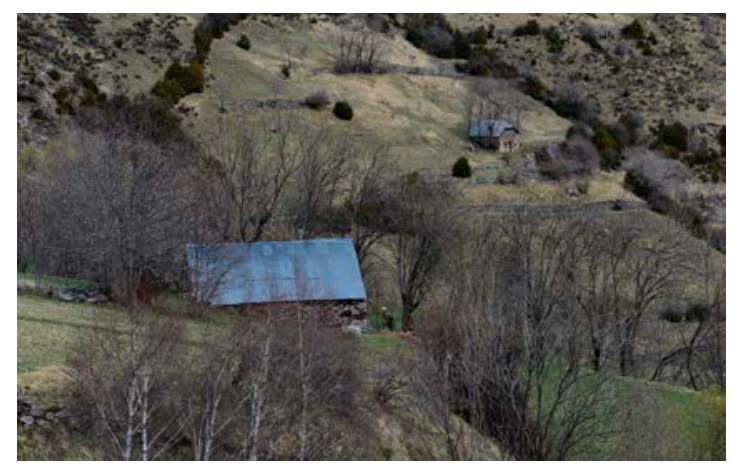

Fuente: Elaboración propia

Fig. 3 Conjunto de bordas en La Poma

Fig. 1 Término Munipal de Gistaín. Esquema de espacios de uso agrícola y ganadero

En el municipio de Gistaín se superponían dos sistemas de explotación ganadera diferenciados. En primer lugar, estaba la cabaña ovina que practicaba una trashumancia descendente, trasladándose durante la estación invernal a distintas localizaciones del valle del Ebro (Binéfar, Monzón, Bajo Cinca y Monegros). Por otro lado, el ganado vacuno, que no trashumaba a Tierra Llana, sino que realizaba un ciclo con recorridos más cortos dentro del mismo municipio, que denominamos trasterminante.

Cada uno de estos tipos de ganado seguía su propio ciclo anual diferenciado, pero no independientes uno de otro, sino que estaban combinados de forma que se conseguía el máximo aprovechamiento de los pastos, asegurando al mismo tiempo su sostenibilidad. Para ello se dispusieron unas normas muy complejas que regulaban todos los aspectos relacionados con los ganados y con la apertura,veda y periodos de uso de los pastos. 


\section{Paisajes agrícolas y ganaderos. Arquitecturas asociadas}

Dentro del conjunto del territorio agrogranadero del municipio de Gistaín es posible destacar varios conjuntos paisajísticos representativos.

En el fondo de valle del Cinqueta y la parte baja de las laderas de los barrancos laterales encontramos una composición compleja, formada por parcelas individuales dedicadas mayoritariamente a prados de siega y cerradas mediante árboles, casi siempre fresnos. Este cierre vegetal cumplía una triple función: por un lado, limitaba la entrada del ganado, restringiéndolo al propio y segregaba el prado de los comunales; por otro, ayudaba a mantener una cierta humedad en el suelo y proporcionaba sombra a los animales en los momentos de máxima insolación; por último, las hojas de los fresnos constituían un forraje adicional para los periodos invernales. Se cortaban las ramas cada cierto número de años, alamacenándose bien en las bordas, bien sobre los mismos árboles para su secado y posterior administración al ganado (Fillat, 1984). Este tipo de paisaje se suele denominar con el término francés bocage, sin traducción directa la castellano (Fig. 2). Es resultado de un proceso de privatización e individualización del espacio pastoral. Está relacionado con el ganado mayor, tanto vacas como animales de labor y mulas de recrío, que permanecían en el valle durante el periodo invernal (Faucher, 1931). En la vertiente española del Pirineo solo aparece en la franja más septentrional, ligado a un régimen de precipitaciones que posibilita el mantenimiento de prados. Aparece en los valles de Gistaín, Bielsa, Benasque o Arán, siendo en cambio inexistentes en otros como los valles de Broto y Vió. Es mucho más común en la vertiente francesa, en la que abundan también las bordas, tipología arquitectónica asociada al prado de siega.

La borda es un edificio de uso mixto, compuesto por dos plantas superpuestas con diferente función. En la planta baja se dispone el establo, dotado con pesebres para albergar al ganado en el periodo invernal o en aquellos días en que no era posible hacerlo pastar en el exterior. En la planta alta, pajar o henil, se almacenaba la hierba procedente de los prados, pero también la paja de los campos de cereal, una vez separado el grano (Fig. 4). Las bordas se relacionan con el ciclo estacional trasterminante del ganado bovino. Cada familia podía disponer de varias de estas construcciones, hasta 6 o 7 en algunos casos, dispuestas de forma escalonada en distancia al pueblo y en altitud. Se distinguen por tanto un primer tipo de bordas, con un uso invernal, situadas en cotas más bajas y próximas al pueblo. En ellas se estabulaban las vacas desde finales de noviembre hasta principios de marzo, atendidas diariamente por los vaqueros que acudían desde las casas. Otras eran empleadas a partir del mes de marzo y hasta mediados de junio. A ellas se trasladaban los animales en cuanto el tiempo lo permitía, para pastar los prados y consumir el heno acumulado en los pajares hasta el momento de subir a los puertos. Esto sucedía hacia el 20 de junio, fecha en que se constituían la vacada comunal y otro conjunto agrupando los animales de labor, denominados mimardos. Estos rebaños se encomendaban a vaqueros contratados para todo el verano. Durante este periodo estival se dejaba crecer la hierba en los prados, para después segarla hacia finales de julio y almacenarla en los pajares de las bordas como reserva de forraje para la siguiente temporada. Este conjunto de prados de siega y bordas intermedias volvía a ser visitado por el ganado al bajar de la montaña, desde mediados de septiembre hasta finales de noviembre, aprovechando el rebrote de la hierba antes de ser retirado a las bordas más bajas (Fig. 3).

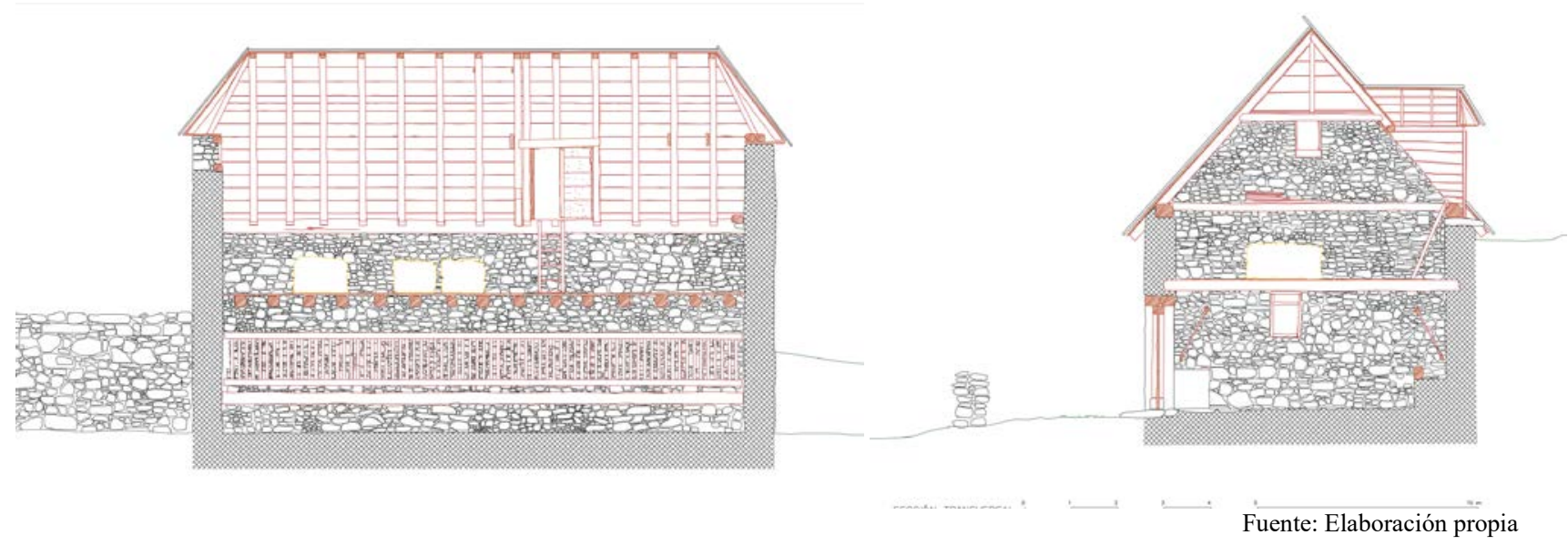

Fig. 4 Sección longitudial y transversal de la borda de casa La Fon en Las Corcillas 
Un segundo paisaje representativo del municipio de Gistaín son los panares. Constituían una particular forma de cultivos en ladera, ya en desuso, con un uso mixto agrario y ganadero en el que se alternaban el cultivo de cereales para autoconsumo y el uso como pastos para el ganado en distintos momentos del ciclo anual (Fernández, 2019). Se trataba de un elaborado ejemplo de cooperación entre el ámbito privado agrícola y la explotación comunal ganadera de los recursos, que se mantuvo activo hasta los años sesenta del pasado siglo. Antiguamente se extendían por amplias zonas de los valles altopirenaicos, pero desaparecieron mucho antes de la vertiente norte. Eran propios de pueblos con términos de elevada altitud media y con un relieve muy accidentado, en los que las escasas posibilidades de aprovechamiento agrícola obligaban a aumentar la superficie dedicada a los cultivos poniendo en producción laderas alejadas en las que unas condiciones de orientación, suelo o disminución de la pendiente ofrecían la más mínima oportunidad (García-Ruiz, 1988, p. 12). En cambio, en valles con fondo amplio y plano de origen glaciar su disposición no fue necesaria, como en Benasque o muchos valles de la vertiente francesa.

Los panares se situán relativamente alejados del núcleo de población y de los campos de cultivo primarios, a altitudes entre los 1300 y los $1900 \mathrm{~m}$. En ellos se cultivaban cereales, fundamentalmente centeno, y también, desde el siglo XVIII, patatas. El sistema de cultivo empleado era de año y vez, con un ciclo bienal en el que se cultivaba la tierra un año y se dejaba en barbecho el siguiente. Esta regla respondía también a un factor puramente climático. Debido al ciclo vegetativo extremadamente lento como resultado de las bajas temperaturas se sembraba el centeno en el mes de agosto y se recogía la cosecha a finales del mismo mes o durante septiembre del año siguiente, demasiado tarde para preparar el suelo para una nueva cosecha (Daumas, 1976, pp. 324-325; Balcells, 1986, p. 166; Lasanta, 1989, pp. 55 y 89). Por esta razón, los panares formaban dos conjuntos, denominados añadas, situados en diferentes zonas que se cultivaban siguiendo un régimen alternativo obligatorio entre ellos, regulado por la costumbre.

En Gistaín existieron cinco panares: Igüerra, la Poma, Viciele, Lisiert y Viadós. Se organizaban en la añada de Viciele, que incluía también el panar de Igüerra y el de Lisiert y la añada de La Poma, que incorporaba también el panar de Viadós, el más lejano respecto a la población, a tres horas y media de distancia. Estos dos conjuntos estaban equilibrados en cuanto a número de campos y parcelas.

Los panares estaban relacionados con el ciclo anual del ganado ovino trashumante, que aportaba el abono orgánico necesario para poner en práctica los cultivos de cereal en unos suelos escasos y pobres. Cuando los rebaños llegaban al valle una vez terminada la invernada en el Valle del Ebro a finales del mes de mayo, sin pasar por el pueblo, acudían directamente a los pastos de tránsito. El periodo que permanecían en ellos se aprovechaba para abonar los panares que se encontraban en barbecho mediante el sistema del redileo. Se encerraban los animales en corrales móviles de madera, barandaus, para pernoctar, aproximadamente desde mediados de mayo hasta finales de julio, cambiando cada noche el corral de lugar para conseguir un estercolado uniforme. Del mismo modo, a la hora de bajar de los puertos, los rebaños acudían a los otros panares, para entonces recién cosechados, aprovechando el rastrojo como pasto. Por ello, las parcelas incluidas dentro de los panares carecían de cierres o divisiones. Para facilitar el tránsito de los animales, las laderas que ocupaban eran aterrazadas por medio de suaves taludes herbosos, denominados espuenas, y no mediante muros. Las parcelas particulares aparecen por tanto sin divisiones de propiedad, con un aspecto continuo que constituye uno de sus más destacados rasgos paisajísticos (Fig. 5).

Asociada a los panares aparece otro tipo de construcción, pequeñas cabañas, cabanas, de planta rectangular o cuadrada, cubiertas a dos aguas con losas de piedra arenisca o pizarras y una superficie útil que oscila entre los 6 y los $15 \mathrm{~m}^{2}$ (Fig. 6). Su función era guardar aperos, los tramos de los corrales móviles para encerrar las ovejas, denominados baranas, y alojar a los pastores en los periodos de primavera y otoño en los que los rebaños pastaban por los alrededores. Eran por tanto arquitecturas de hábitat exclusivamente humano, no incorporando espacios destinados a los animales ni tampoco pajar para almacenar forrajes. En muchas de estas cabañas una prolongación lateral de uno de los muros servía para disponer un pasillo para el ordeño de las ovejas, colocando una serie de baranas en paralelo. Existen también pasillos de ordeño construidos enteramente con muros de piedra seca de aproximadamente 1,5 $\mathrm{m}$ de altura, denominados corrales de muyir. Pueden llegar a los $40 \mathrm{~m}$ de longitud, por una anchura que no llega a los $3 \mathrm{~m}$, abiertos solo por uno de sus extremos. En ellos se introducía todo el rebaño, situándose los ordeñadores en la salida, sacando los animales según se iban ordeñando. Este tipo de construcciones no son comunes en la comarca de Sobrarbe, donde los más conocidos son los situados en Escartín, en el Sobrepuerto. 
Dentro de los espacios ya de estricto uso ganadero, los pastos intermedios o de tránsito y los puertos se caracterizan por estar constituidos por amplios pastizales, con muy poco o ningún arbolado. En ellos la presencia de las actividades humanas es estacional, y por tanto la transformación antrópica es en apariencia más tenue que en las zonas de uso agrícola. Sin embargo, la existencia de estas áreas de pasto se debe en gran medida a la acción humana a través de siglos de ganadería. La extesión de las áreas pastables fue incrementada históricamente para alimentar a los rebaños, rebajando el límite superior del bosque. Su uso estaba regulado por las ordinaciones de la montaña, un conjunto de normas que establecían las fechas en que se podía introducir los animales en ellos y los periodos en que estaban vedados.

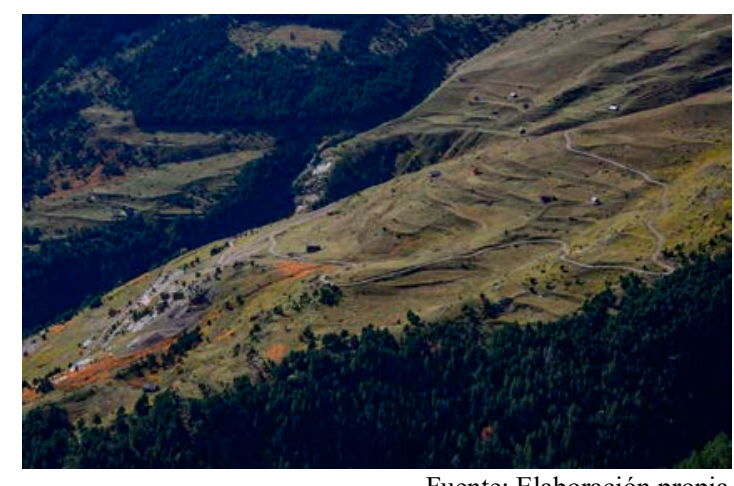

Fig. 5 Panar de Viciele
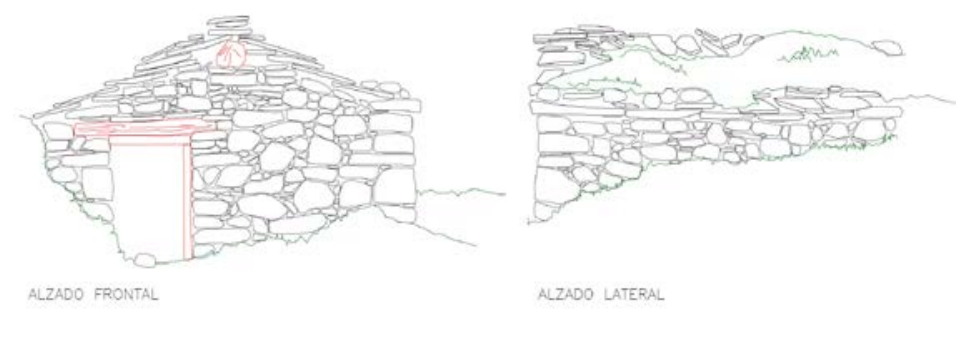

Fuente: Elaboración propia Fig. 6 Cabana de casa Ferrer, panar de La Poma

Los puertos del municipio de Gistaín ocupan las grandes extensiones de montaña por encima de los $1800 \mathrm{~m}$ de altitud situadas en el sector norte del municipio. Su aprovechamiento era realizado mediante rebaños comunales, tanto de ganado ovino, como de vacuno. A principios de siglo XX se formaban dos compañías para subir el ganado lanar a los puertos, cada una de ellas dividida a su vez en dos rebaños de en torno a 1500 cabezas cada uno. El espacio de pastos quedaba dividido por el curso del Cinqueta de la Pez, de forma que una compañía recorría los puertos o montañas situados en su lado oriental y la otra los del lado occidental (Fig. 1). Cada uno de los rebaños tenía destinadas una serie de partidas, que recorría de forma sucesiva, permaneciendo un periodo de entre unos pocos días a poco más de una semana en cada una de ellas. De esta forma de aprovechamiento deriva la huella antrópica en la montaña, consistente en los vestigios de las antiguas cabañas de los pastores en las pletas, los lugares en los que pernoctaba el rebaño, generalmente sir cercar. A diferencia de lo que sucede en otras áreas de montaña, donde la orientación económica del ganado ovino era hacia la producción de leche y la elaboración de quesos, en la montaña de Gistaín no encontramos instalaciones con una carácter fijo o estable. El ordeño y la fabricación de quesos requieren una serie de instalaciones más complejas y la permanencia más prolongada en un lugar de la montaña, que se convierte en la base de todos los recorridos diarios. El modelo ganadero chistabino estaba en cambio orientado a la obtención de lana y de carne. Este tipo de ganado tiene una mayor movilidad, al no tener que ser ordeñado mañana y tarde, que era aprovechada para hacerlo dormir en distintas localizaciones con objeto de abonar mediante sus deposiciones y mejorar así el pasto, de forma similar al método empleado en las parcelas agrícolas. Este era un aspecto que estaba incorporado en las normas comunitarias, que establecía la obligación para cada una de las compañías de cambiar las pletas de lugar cada dos años, precisamente para mejorar la calidad de la hierba, y se establecían las correspondientes multas en caso de no cumplirse este requisito. El cambio de pleta implicaba la construcción de una nueva cabaña, que por los pocos días en que era usada, y su carácter efímero al tener que ser trasladada cada dos años, eran construcciones muy rudimentarias y sencillas. Además, era muy habitual reutilizar los materiales de las cabañas anteriores a la hora de levantar las nuevas. Como consecuencia encontramos dispersos por la montaña una gran cantidad de restos de cabañas, casi siempre reducidos a los alineamientos de piedras de su fundación en el suelo, pues el resto de los muros era reempleado en otro lugar cercano (Figs. 7 y 8 ). 


\section{Fernández Piñar}

\section{Conclusiones}

Las áreas de montaña constituyen paisajes profundamente transformados por el hombre desde tiempos muy antiguos. Las huellas de las distintas sociedades que lo han habitado y de las diferentes actividades que sobre ellos se han desarrollado se encuentran presentes en el paisaje, que podemos entender como una serie de capas superpuestas a modo de palimpsesto.

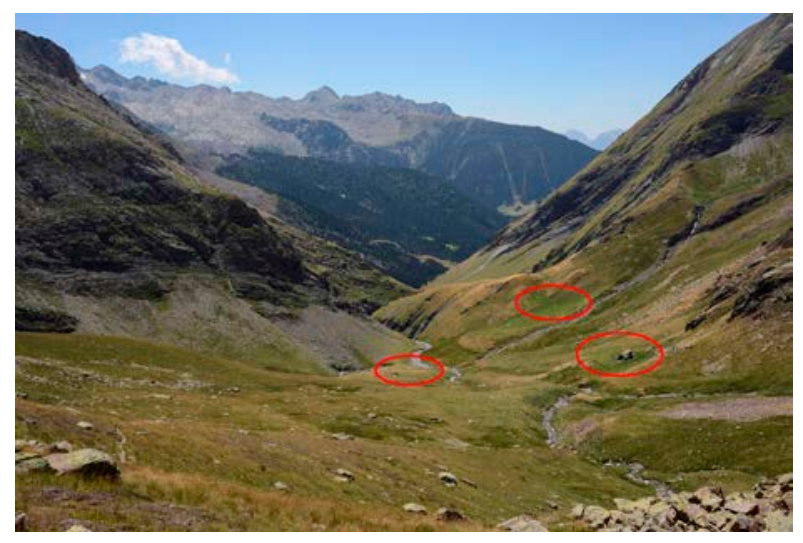

Fuente: Elaboración propia

Fig. 7 Paisaje del Puerto de Añes Cruzes. Se señala la situación de varias pletas con sus cabañas de pastor

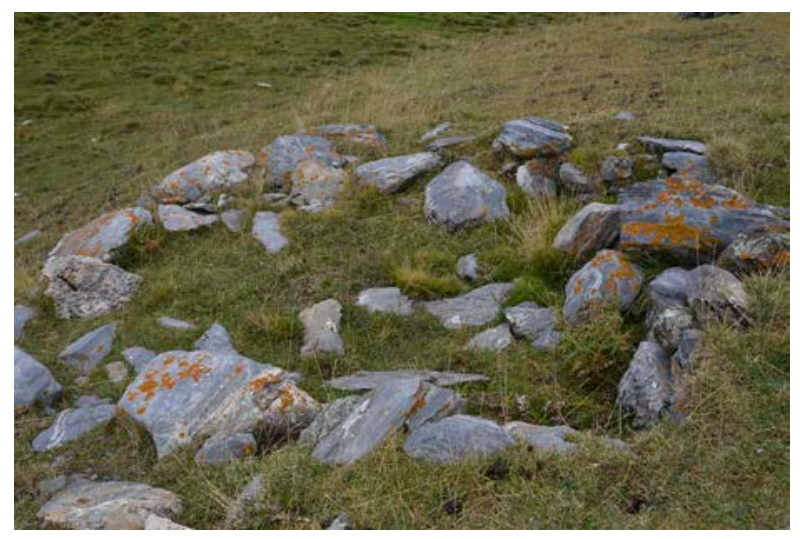

Fuente: Elaboración propia Fig. 8 Huella de cabana pastoril en Loriele

Desde esta forma de entender el paisaje podemos hacer una lectura diacrónica de las transformaciones que el territorio ha sufrido producto de la acción antrópica. Dentro de estas transformaciones, una parte importante de la construcción del paisaje humanizado se debe a la cantidad y diversidad de pequeñas arquitecturas auxiliares de las actividades agrícola y ganadera dispuestas sobre el territorio. Cada una de estas arquitecturas tiene una tipología y un sistema constructivo adaptado a su situación y a su función dentro de los sistemas de organización agrícola y pastoril.

Resulta complicado datar este tipo de construcciones, pues son muy infrecuentes las referencias documentales a ellas. Se ha localizado la mención a una borda en un documento de 1626, aunque muy probablemente existieran ya con anterioridad. La generalización de los estudios paleoambientales en los últimos años ha aportado nuevos datos. El estudio de la secuencia polínica extraída de los sedimentos de la Basa de la Mora (Pérez-Sanz et al., 2011), situada a escasa distancia de Gistaín dentro del mismo valle, sugiere una intensificación de las actividades humanas a partir del siglo XIV. La presencia de polen de fresno, árbol introducido por el hombre asociado al sistema prado de siega-borda, permite proponer en este periodo el origen de estas edificaciones. Por otro lado, el estudio detallado de los amillaramientos del siglo XIX constata un importante incremento en su número, así como la reforma y ampliación de las existentes, en el último tercio del siglo XIX y el primero del XX, seguramente asociado al negocio del recrío de mulas. Estas se compraban en Francia jóvenes, se criaban y domaban durante dos años y se vendían posteriormente en las ferias de ganado para los trabajos agrícolas y el transporte. Solo a partir de la segunda mitad del siglo XX aumentó considerablemente el ganado vacuno, hasta entonces escaso y dedicado fundamentalmente al trabajo, al tiempo que desaparecieron las mulas como consecuencia de la mecanización. En la actualidad el descenso del número de ganaderos ha provocado el abandono de muchas bordas, y solo algunas pocas han sido reformadas para usos turísticos.

Las cabanas asociadas a los panares no aparecen en ningún registro documental, pero podemos situar su origen en el mismo momento en que se ponen en cultivo estos espacios. Existen menciones a los panares chistabinos al menos desde 1555. Su implantación fue probablemente anterior, como consecuencia del incremento poblacional y el desarrollo de los grandes rebaños trashumantes. La necesidad al mismo tiempo de más espacios de cultivo para la alimentación humana y de más pastos para el ganado se solucionó mediante las normas de uso de los panares, que permitían satisfacer ambas, manteniendo el uso como pastos comunales y al tiempo asegurando el abono imprescindible para los cultivos. Solo con la definitiva crisis de la trashumancia y el abandono de los cultivos de cereal en el último tercio del siglo XX dejaron de emplearse las cabanas, que en la actualidad presentan en su mayoría un estado de ruina. 
En cuanto a las cabanas pastoriles en los puertos, su datación es imposible sin el recurso a excavaciones arqueológicas. En entornos cercanos del Pirineo se han documentado restos que se remontan hasta el Neolítico (Gassiot, 2016). La distibución espacial de las estructuras inventariadas sugiere una sucesión de distintos sistemas ganaderos, siendo la mayor parte de los restos correspondientes a los grandes rebaños comunales trashumantes. En la actualidad no tienen uso, y muy pocas permanecen en pie.

En conclusión, en el municipio de Gistaín es posible comprobar hasta que punto la presencia de las construcciones agroganaderas se extiende por todo el territorio, con distintas formas e intensidades, pero abarcando desde las proximidades del núcleo de población hasta las cabañas de pastores en los puntos más alejados del municipio (Fig. 9).

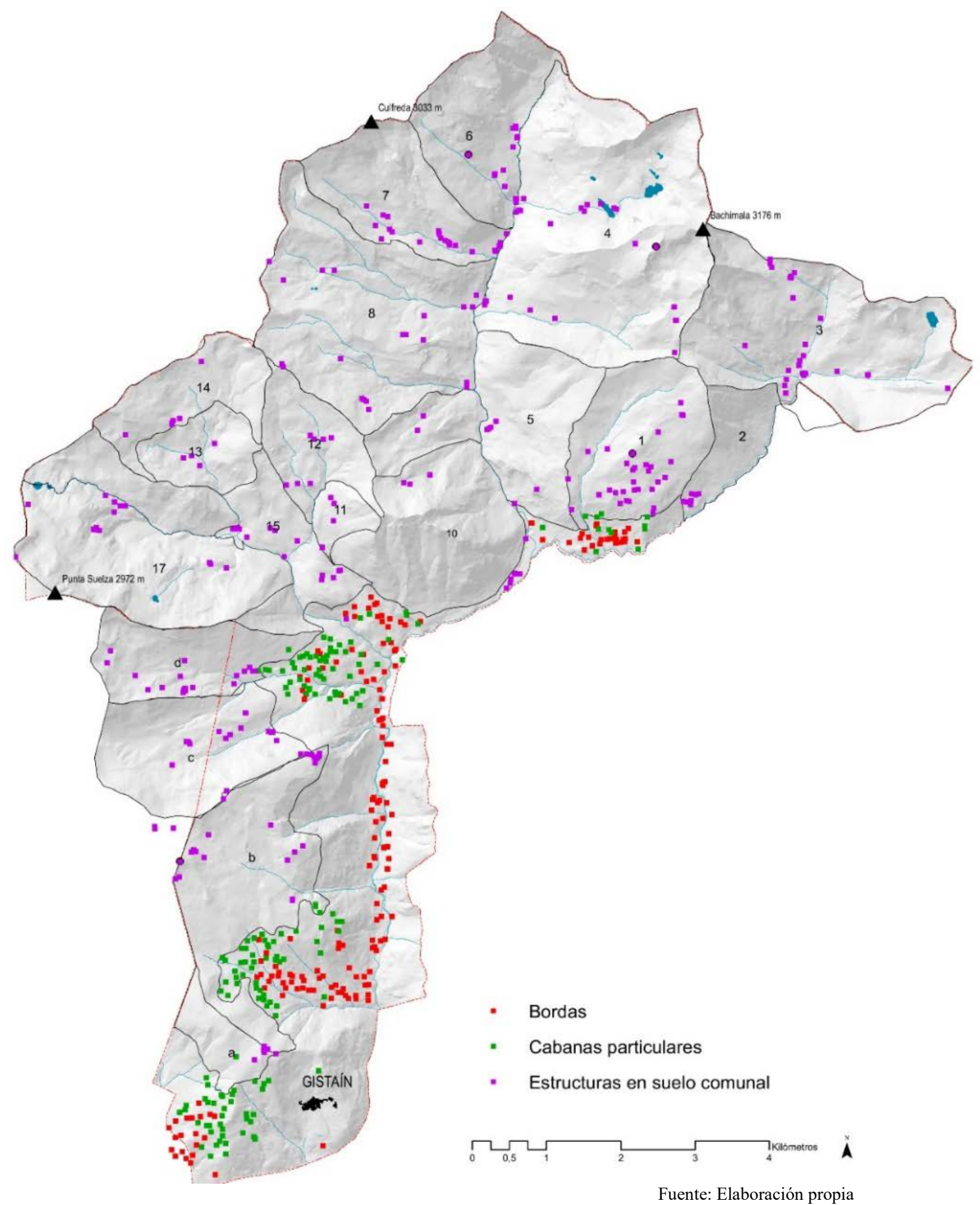

Fig. 9 Término Munipal de Gistaín. Localización de las distintas construcciones 


\section{Fernández Piñar}

\section{Referencias}

Balcells, E., y Creus-Novau, J. (1986). Reflexiones sobre los límites altitudinales de las residencias humanas permanentes en el Alto Pirineo aragonés. Pirineos, 127, 153-74. Recuperado de https://digital.csic.es/handle/10261/95397.

Daumas, M. (1976). La Vie rurale dans le Haut Aragon oriental. Madrid: CSIC.

Faucher, D. (1931). Le bocage pyrénéen. Revue géographique des Pyrénées et du Sud-Ouest, 2:3, 362-65. DOI: https://doi.org/10.3406/rgpso.1931.4024.

Fernández, C. (2019). Origen, localización y evolución de los panares del municipio de Gistaín. Historia agraria: Revista de agricultura e historia rural, 78, 67-97. Recuperado de https://dialnet.unirioja.es/servlet/articulo?codigo=6973753.

Fillat, F., Gómez, D., y Creus-Novau, J. (1984). El fresno de hoja ancha como árbol semi-salvaje en el Pirineo de Huesca. Acta Biologica Montana, 4, 445-54. Recuperado de http://digital.csic.es/handle/10261/108390.

García-Ruiz, J. M. (1988). La evolución de la agricultura de montaña y sus efectos sobre la dinámica del paisaje. Revista de Estudios Agrosociales, 146, 7-37. Recuperado de https://digital.csic.es/handle/10261/100832.

Gassiot, E. (Ed.). (2016). Arqueología del pastoralismo en el Parque Nacional d'Aigëstortes i Estany de Sant Maurici. Montañas humanizadas. Madrid: Organismo Autónomo Parques Nacionales.

Lasanta, T. (1989). Evolución reciente de la agricultura de montaña: el Pirineo aragonés. Logroño: Geoforma Ediciones.

Pérez-Sanz, A., González-Sampériz, P., Valero-Garcés, B. L., Moreno, A., Morellón, M., Sancho, C., Belmonte, A., Gil-Romera, G., Sevilla, M., y Navas, A. (2011). Clima y actividades humanas en la dinámica de la vegetacion durante los últimos 2000 años en el Pirineo central: el registro palinológico de la Basa de La Mora (Macizo de Cotiella). Zubia, 23, 17-38.

Rey, J. (2014). El final de la Prehistoria en Sobrarbe. En E. Gassiot, I. Clemente y J. Rey (Eds.), Sobrarbe antes de Sobrarbe. Pinceladas de Historia de los Pirineos (pp. 71-93). Huesca: Centro de Estudios de Sobrarbe. 